\title{
Estimating PCI Using Vibration Data for Asphalt Concrete Pavements
}

\author{
Ufuk Kırbaş ${ }^{1}$, Mustafa Karaşahin ${ }^{2}$ \\ ${ }^{1}$ Ondokuz Mayis University, Department of Civil Engineering, Kurupelit Campus, \\ Samsun 55139, Turkey \\ ${ }^{2}$ Istanbul University, Department of Civil Engineering, Avc1lar Campus, \\ Istanbul 34320, Turkey \\ ufuk.kirbas@omu.edu.tr; mkarasahin@istanbul.edu.tr
}

\begin{abstract}
The pavement condition index (PCI) provides an indication of the current performance of a pavement, and takes the form of a numerical rating, with 0 being the worst possible condition and 100 being the best. The PCI is a subjective method for the evaluation of pavements that is based on inspection and observation. Knowledgeable and experienced pavement engineers make a systematic evaluation of road conditions by driving on the road network, and enter their observations into a database for further evaluation. This case study made use of PAVER software to obtain PCI values for twenty road sections that experience different volumes of traffic in the Samsun region. First, the condition of each road section was evaluated using the PAVER procedure, after which, vertical vibration data was recorded for the same road sections using accelerometers mounted at three different locations within the car: the driver's seat, over the central axis and the front passenger seat. The car was driven at a constant $40 \mathrm{~km} / \mathrm{h}$ during the tests. In the final stage, the relationship between the vibration data and the PCI values obtained during the site study was examined, and PCI prediction models were created for use in the assessment of urban roads.
\end{abstract}

Keywords: PCI, pavement performance, pavement management system, vibration, vertical acceleration

\section{Introduction}

Pavements are the most important infrastructural element, providing comfort and security to users of the road, whether they are drivers or passengers. There is an obvious need to establish systems for the management of pavement conditions, which are vital importance for the lives of drivers and passengers, so as to identify any important investments that need to be made. Pavement management, in the widest sense, refers to the work programme that oversees all processes related to pavements, and includes planning, programming, designing, construction, maintenance, repair and renovation. A pavement management system (PMS) facilitates the configuration of the tools and methods that will be used to determine the most suitable maintenance and the repair calendar for decision makers for a given period of time [1].

The PMS concept that has been in place since the 1950s has been adapted over time in line with new innovations and technological developments. In the 1990s in particular, several books and research reports were published explaining the theory of the PMS concept, its levels, and its data collection and assessment techniques. This period also saw the publication of a PMS standard, known as the American Society Testing and Materials (ASTM) E1889-97 [2]. Publications explaining the theory of the PMS concept and its components provide information on the laws to which related institutions are subject, the principals used in the separation of sections in the evaluation of the road network, the data that should be included in the PMS database, methods of data collection and processing, methods and required frequency of data gathering, deterioration forecast models, predictions for the future, restoration strategies and determination techniques. These studies have shown that the most problematic part of the PMS system is in the determination of current performance [1, 3-7].

Previous literature contains a number of PMS studies related to urban roads, including specific studies of such cities as Dammam (Saudi Arabia) and Lisbon (Portugal), as well as several in the United States, in America Bowling Green (Kentucky), Chittenden (Vermont), Dublin (Ohio), Folsom (California), Los Angeles (California), Marion (Florida), Philadelphia (Pennsylvania) and Tinley Park (Illinois). In these studies, it can be seen that for the determination of current pavement performances, it is generally distress data of the pavement surfaces that is used. The predominant indexes used were the pavement condition index (PCI), the pavement condition ratio (PCR), the pavement surface evaluation and rating system (PASER) and the remaining service life (RSL) index [6, 8-10]. 
Another subject on which the researchers focused in these studies was the establishment of models based on an analysis of the relationships between the data. Generally, researchers focus on models that can evaluate the broad range of surface evaluation data related to cracks and roughness. It is apparent that these models were created using of advanced mathematical modelling techniques, such as artificial natural works, physiologic genetic algorithms, data mining, ant colony, etc. In the subject of this study, it can clearly be seen that the most interesting topics are those that show the relationship between pavement cracks and International Roughness Index (IRI) [11-17].

It is known that the most difficult stage is determining the current performance of the pavements in a system set up to provide the management of pavements. In many studies it has been determined that there is a strong relationship between the structural performance of a pavement and pavement roughness [1]

There are several studies in literature aimed at determining pavement roughness through an analysis of the relationship between the current performance of a pavement and the vibrations recorded using accelerometers [18-20]. It has been observed in these publications mostly on the vehicle dynamics that the researchers have used acceleration data as an independent variable in vehicle dynamics modelling or in researching the correlations with pavement roughness data. It was seen as the ultimate point reached in the PMS engineering on the evaluation of vibrations is the generation of the simulation models and the real-time test mechanisms. In other words, when all the PMS studies are evaluated, it can clearly be seen that, pavement serviceability can be determined even before the road is constructed due to the deteriorations in the pavement, which will be produced following its design by using the latest technology, that will occur in time and the impact of these deteriorations on the drivers [18, 21-26].

Pavement unevenness results in significant negative road-vehicle dynamic interactions, affecting ride quality and also facilitating vehicle damage and pavement distress. The results obtained in previous studies related to weighted vertical acceleration $\left(a_{w z}\right.$, that is, the WBV index, according to ISO 2631-1 standard), show good correlations ( $\mathrm{R}^{2}$ from 0,75 to 0,93 , depending on vehicle speed) with the IRI values of the examined road sections [19]. It should be noted that the relationship between the PCI and vertical acceleration has to date not been studied in literature.

It is understood from the literature, the most difficult step in the establishment of a PMS to determine the current performance of pavements. PCI is widely used especially in evaluating the performance of pavements of urban roads. In this study were investigated relationships between PCI and vertical vibrations occur in the vehicle. PCI prediction models were created for use in the assessment of urban roads.

\section{PAVER System and Pavement Current Performance}

The PAVER system is an evaluation procedure for the assessment of the current performance of the pavement by evaluating data gathered according to the identification guide with the code ASTM D 6433-11 in sample areas of $225 \pm$ $90 \mathrm{~m}^{2}\left(2500 \pm 1000 \mathrm{ft}^{2}\right)$, chosen randomly in line with distribution principals. The evaluation is made by reading the resulting values from the deduct value tables, which were prepared in accordance to the deterioration type with the help of the value derived by proportioning the surface deterioration data that were gathered at the low (L), medium (M) and high $(\mathrm{H})$ severity levels to the size of the sample unit. Extracting the obtained deduct values from 100 results in a PCI value, which defines the performance of the pavement with a numerical value of $0-100$. A PCI value of 100 denotes a pavement on which there is no distress and which is in the best condition, while a 0 value indicates a pavement that is completely corrupted and cannot be used. The PCI is an index that shows the current performance of a pavement, and is based on an evaluation of the common components of the distress type, distress quantity and distress severity [3, 27], as shown in Figure 1. 

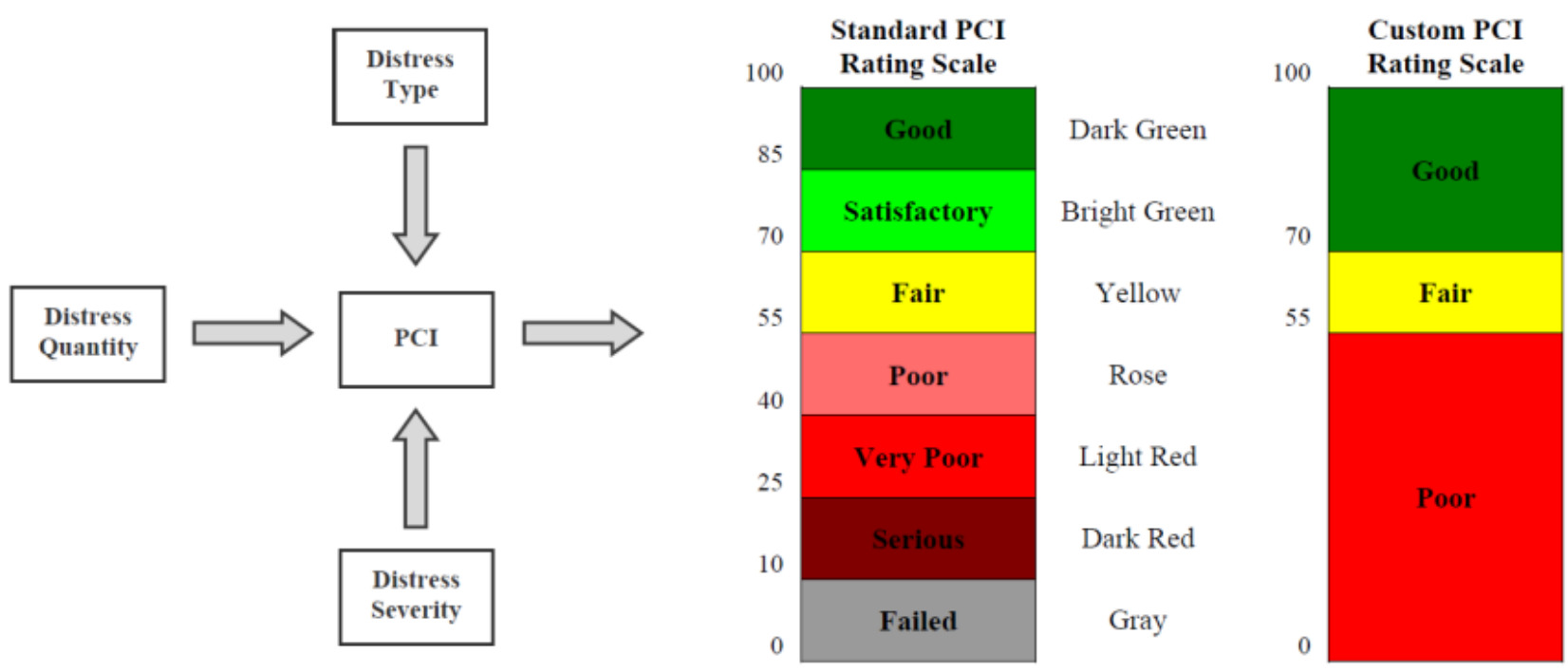

Fig.1: Pavement Condition Index (PCI) ranges [27].

In the PAVER system, the rating scale concept used to describe the performance of the pavement is used in the description of the current status with different colors. Simultaneously, pavement performance was also defined as 'good' or 'satisfactory' with verbal expression in this PCI rating scale. With the PAVER system used as a base, the distress classification used for hot mix asphalt (HMA) coated roads and parking lots - defined in the standard ASTM D 6433-11 code.

Pavement distress data of 20 different urban highways in 98 different sections in the Turkish city of Samsun was obtained, as defined in the ASTM D 6433-11 standard. The data covers a total $55750 \mathrm{~m}$ length of road with a total area of $337867 \mathrm{~m}^{2}$. The data was evaluated to identify the current performance of the pavement according to the PAVER system. From the roads selected for the surface condition evaluation, 1415 sample units were first identified, of which 1375 were analysed for the collection of surface data. Besides gathering surface corruption data for 97.2 percent of the originally identified areas, all investigated areas were subjected to surface distress evaluations, ensuring the precision of the project according to the requirements of the PAVER system.

Causes of pavement distresses are load, climate and others in the PAVER system. Distresses caused by the load (alligator cracking, edge cracking, potholes, rutting) and others (bumps and sags, corrugation, depression, lane/shoulder drop-off, bleeding, patching, polished aggregate, railroad crossings, shoving, slippage cracking, swelling) are to be considered the increase in the vertical vibration and consequently the reduction of the driving comfort. Climate-induced distresses (block cracking, reflection cracking, longitudinal and transverse cracking, ravelling, weathering) is not caused by too much in the formation of vertical vibration. In the analysed network, load induced distress of $33 \%$, other induced distress of $59 \%$ and distresses caused by climate is $8 \%$. Therefore, it is obvious that principally vertical vibration measurements reflects pavement distresses in the network. Mainly pavement distresses seen that alligator cracking (18.5\%), corrugation (11.8\%), depression (15.2\%), patching (19.5\%) and potholes (13\%).

\section{Vibration Evaluation}

There is a well-known high correlation between the present serviceability index (PSI) (reflecting driving comfort; used to determine the current performance of the pavement) and surface roughness $[1,21]$.

In the present study, the surface roughness component was evaluated through an analysis of acceleration data, recorded in a vertical direction inside the car. The evaluation was made according to the ISO 2631-1 'Mechanical vibration and shock - Evaluation of human exposure to whole-body vibration' standard, which calls for data from the 3D accelerometer measurement to be collected different positions (sitting, standing and lying supine) and defines the calculation principals of the evaluation parameters $a_{w}$ (weighted root mean square), MTVV (the maximum transient vibration value) and $V D V$ (the fourth power vibration dose value) [28]. In order to evaluate whole body vibration following the definitions specified in the ISO 2631-1 standard, it is necessary to place the accelerometer right under the driver for the purpose of providing the interaction with the passenger. The ISO 2631-1 standard advises that the vibration signals should be regulated through the use of the butterworth filtering technique according to the $1 / 3$ octave band frequency. Following the measurements, by applying the gains defined in the frequency weight filters to the 
acceleration values that were gained in the $1 / 3$ octave bands, the weighted total values $\left(a_{w}\right)$ in the related axis direction can be obtained from the equations provided below.

By multiplying the acceleration frequencies, separated according to the $1 / 3$ octave band frequencies, with the weights defined in the standard, the acceleration values can be found. According to the ISO 2631-1 standard, the $a_{w}$ component is considered the most suitable parameter in explaining the acceleration transferred, thus felt by the effected person.

$$
a_{w}=\left[\sum_{i}\left(w_{i} a_{i}\right)^{2}\right]^{\frac{1}{2}}
$$

Where;

$a_{w}:$ the configured frequency acceleration ,

$w_{i}$ : the weight factor that was defined to the related factor,

$a_{i}$ : the rms value for $1 / 3$ octave band interval [28].

In some specific short time periods, by evaluating all of the acceleration values recorded in over a determined time interval, the random shock vibrations that are transferred to the passengers can be defined. The MTVV parameter is produced by determining the maximum weighted squared average for a determined period of time $(a w(t 0))$. The related moving squared average value is defined as;

$$
a_{w}\left(t_{0}\right)=\left\{\frac{1}{\tau} \int_{t_{0}-\tau}^{t_{0}}\left[a_{w}(t)\right]^{2} d t\right\}^{\frac{1}{2}}
$$

Where;

$a_{w}(t)$ : instant frequency weighted acceleration value,

$\tau$ : average moving time period,

$t$ : variable measurement,

$t_{0}$ : the period of measurement (ISO 2631-1 Standard).

The ISO 2631-1 standard advises "one second" be the time chosen for the time period of the moving average, which leads to the calculation of the maximum transient vibration value as below [28].

$$
M T V V=\max \left[a_{w}\left(t_{0}\right)\right]
$$

Since the $V D V$ method is an outcome reflecting the total vibration value that an individual was exposed rather than the average value, it has not been included in the evaluation.

\section{Case Study}

To evaluate the effects of distresses in the pavement, the vertical vibration value (acceleration value) of a moving car measuring between 4100-4600 $\mathrm{mm}$ was taken, corresponding to a lower middle class $\mathrm{C}$ segment according to the Euro Car classification. Using a vibration measurement set, comprising 3 pcs accelerometers, designed for the measurement of vibration $( \pm 4 \mathrm{~g}$, sensitivity $500 \pm 15 \mathrm{mV} / \mathrm{g})$, one GPS antenna $(<15 \mathrm{~m}$ accuracy $)$ and one datalogger, the vibration values in a vertical direction were recorded on the roads on which there a PAVER evaluation had been made.

Vertical acceleration data and GPS data were collected and transferred to the computer instantly as 1000 pcs $(1000 \mathrm{~Hz})$ per second and as 1 location and 1 speed per second respectively. Accelerometer was placed in three different points during the measurements: Under the driver seat (with the driver), under the front seat (without a passenger) and over the central axis (at the foot level). Following the directions of the ISO 2631-1 standard, an accelerometer was placed in an elastic pad just under the driver, while the others were fixed at their respective positions in order to evaluate the effect to the passenger. The locations of the accelerometers are shown in Figure 2.

The vertical vibration measurements in the $G$ unit provided by the device were evaluated after being converted into the $\mathrm{m} / \mathrm{s}^{2}$ format. As the purpose of this research is to identify the relationship between vibration values and pavement distress, it is apparent that it is necessary to evaluate the profile of the road longitudinally from the measurements. For this reason, during the vibration measurements, as a general principal, the principals of longitudinal profile measurement defined in the ASTM E950, E1082 - 90, E 1926-08 etc. standards were accepted. Following the general 
approach of the related standards, the measurements were taken on the same lane at a stable speed (minimum $20 \mathrm{~km} / \mathrm{h}$ ), being accepted as the criteria to be followed for all measurements.

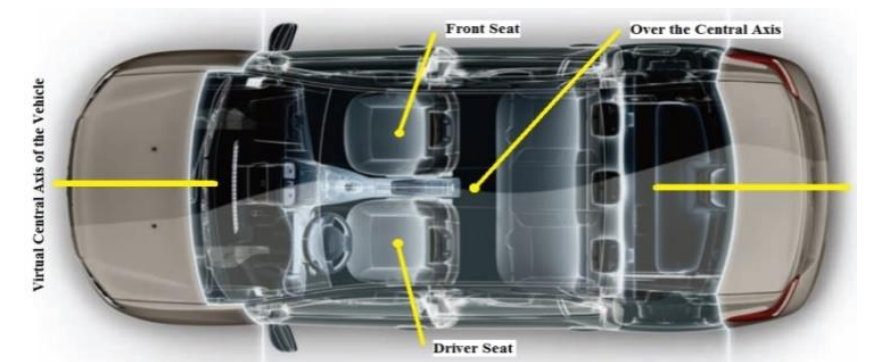

Fig. 2: The position of accelerometers placed inside the vehicle (fig.www.renault.com).

\subsection{Calibration of Vibration Data}

According to the ISO 2631-1 standard accepted as the basis for the evaluation of vibration data, it is known that the $a_{w}$ variable generally reflects the vertical vibration characteristic in any section. Besides, the most appropriate point to the description of the measurement point accepted in the standard is the measurement made on driver's seat. In particular, the mechanical structure of the vehicle and the surface distress on the road on which the vehicle is travelling were determined as the main causes of vertical axis vibration [18].

To rule out the effects of vertical vibrations that occur due to the mechanical structure of the vehicle, calibration parameters were defined. The calibration was made on an HMA covered road with a PCI value (indicating the current performance of the pavement) of 100, on a geometrically aligned section and with a very low longitudinal slope.

The main purpose in the production of calibration parameters is to determine the vertical vibration values that may presumed to be sourced from the mechanical structure of the vehicle and would be expected to occur under all conditions. It is thus accepted that by subtracting the parametric vibration values identified during the calibration from the parametric vibration values found during the measurement of any road, the corrected vibration values will be identified. Below is the equation used for the evaluation of the corr. $a_{w}$ parameter:

$$
\text { Corrected } a_{w}\left(\text { corr. } a_{w}\right)=a_{w} \text { raw }-a_{w} \text { calibration }
$$

The vertical vibration parameter values $\left(\right.$ corr. $\left.a_{w}\right)$ were calibrated by subtracting the calibration values produced to determine the most suitable speed from the vibration values measured during the calibration road evaluation phase. Following the evaluations to determine the corrected values, the relationship between the vertical vibration parameters (corr. $a_{w}$ ) and the PCI data was investigated, beginning with an analysis of the correlations.

During the vibration measurements made at the driver's seat, the weight of the driver was evaluated to identify whether or not it affected the results. To this end, vibration measurements were made on a pilot evaluation road whose roughness characteristics had been accepted as homogeneous and on which calibration measurements had been made, with driver weights of $58 \mathrm{~kg}, 80 \mathrm{~kg}$ and $114 \mathrm{~kg}$ and at speed of $40 \mathrm{~km} / \mathrm{h}$.

These obtained vibration values were evaluated either through practical statistic methods or non-practical statistic methods. The non-parametric statistical evaluation method and the statistic expressiveness values (p) were found using the Kruskal-Wallis H-test, the results of which can be seen in Table 1. In the statistical evaluation, statistically insignificant situations in which the differences between the vibration parameters for different driver weights proved in H1 hypothesis, i.e. $95 \%$ reliability sensitivity, were shown in the shaded areas.

Table 1: Kruskal-Wallis $\mathrm{H}$ test statistical significance values.

\begin{tabular}{|c|c|c|c|c|c|c|}
\hline \multirow{2}{*}{$\begin{array}{c}\text { Speed } \\
(\mathbf{k m} / \mathbf{h})\end{array}$} & \multicolumn{2}{|c|}{ Driver Seat } & \multicolumn{2}{c|}{ Over the Central Axis } & \multicolumn{2}{c|}{ Front Seat } \\
\cline { 2 - 7 } & $\mathbf{a}_{\mathbf{w}}$ & MTVV & $\mathbf{a}_{\mathbf{w}}$ & MTVV & $\mathbf{a}_{\mathbf{w}}$ & MTVV \\
\hline $\mathbf{4 0}$ & 0.056 & 0.190 & 0.085 & 0.118 & 0.000 & 0.000 \\
\hline
\end{tabular}

In a one-sample Kolmogorov-Smirnov test, it was determined that the vibration parameters observed at all measurement speeds and with different weights of driver were appropriate for a normal distribution. The statistical significance values found from the parametric methods using an ANOVA test are seen in Table 2. In the statistical evaluation, statistically insignificant situations in which the differences between the vibration parameters for different driver weights proved in $\mathrm{H} 1$ hypothesis, i.e. $95 \%$ reliability sensitivity, were shown in the shaded areas. 
Table 2: ANOVA test statistical significance values.

\begin{tabular}{|c|c|c|c|c|c|c|}
\hline \multirow{2}{*}{$\begin{array}{c}\text { Speed } \\
(\mathbf{k m} / \mathbf{h})\end{array}$} & \multicolumn{2}{|c|}{ Driver Seat } & \multicolumn{2}{c|}{ Over the Central Axis } & \multicolumn{2}{c|}{ Front Seat } \\
\cline { 2 - 7 } & $\mathbf{a}_{\mathbf{w}}$ & MTVV & $\mathbf{a}_{\mathbf{w}}$ & MTVV & $\mathbf{a}_{\mathbf{w}}$ & MTVV \\
\hline $\mathbf{4 0}$ & 0.051 & 0.079 & 0.065 & 0.083 & 0.000 & 0.000 \\
\hline
\end{tabular}

After the evaluations, it was determined that, there was no significant statistical difference between the vibration values produced in the measurements at the 95 percent reliability level, carried out with drivers weighing $58 \mathrm{~kg}, 80 \mathrm{~kg}$ and $113 \mathrm{~kg}$ and at speed of $40 \mathrm{~km} / \mathrm{h}$. Again with these statistical assessments, the differences between the vibration parameters obtained from the front seat measurements have been found statistically meaningful both with parametric and non-parametric evaluations. Since the differences between the vibration parameters found by the front seat measurements made on a pavement which is in a pretty good condition with minimum vertical vibration values $(\mathrm{PCI}=$ 100) were considered statistically meaningful, the measurements made at this point were accepted as an indicator that these measurements were unreliable in this study.

For this reason, when creating the vertical vibration PCI forecast models (from the main outputs of the study), the measurements made at the front seat position were not included in the evaluation.

\subsection{Pavement Assessments}

After accepting a measurement speed of $40 \mathrm{~km} / \mathrm{h}$ following various evaluations, vertical acceleration measurements were made at the the driver's seat position and over the central axis (for each traffic lane) on 20 highways in 98 sections with PCI values calculated according to the PAVER system. The measurements were evaluated according to the principles of the ISO 2631-1 standard and corrected with the calibration values. The vibration value of each section was found by taking the arithmetic average of the corrected vibration values found for each traffic lane.

In the study, it was found that in urban traffic conditions, it is not possible to drive at a specific speed on a traffic lane, due to a number of factors. For this reason, in the evaluations, the measurement speed was accepted as $40 \pm 5$ $\mathrm{km} / \mathrm{h}$, by adopting $\pm 5 \mathrm{~km} / \mathrm{h}$ as the value of deviation limit. To investigate the changes in measurement speed, measurement speeds were recorded at intervals of one second using the GPS antenna on the device. In the evaluation, the average of instant speed data was taken as the average speed of concerned section and the relevant speed components with unit of $\mathrm{km} / \mathrm{h}$ were obtained for all the measured sections. In the evaluation of the speed component together with the corr. $a_{w}$, the most suitable relationship was found by taking the equivalent on the logarithm base e and dividing the values of corr. $a_{w}$. The relative equation is presented below:

$$
\text { corr. } a_{w} / \ln (\text { Speed })
$$

The linear relationship between the PCI values and the corr. $a_{w}$ values were found to be very high, and it was determined that the highest correlation was at the driver's seat position, followed in turn by over the central axis and at the front passenger seat. Also it was seen that including the speed component in the evaluation caused the correlation to increase slightly. In the evaluation made using the SPSS program, the correlations were found to be meaningful with a 95 percent reliability sensitivity rate. The correlation values that were found following the evaluations are presented in Table 3 .

Table 3: Vibration - PCI linear correlation relationship.

\begin{tabular}{|l|c|c|}
\hline & corr. $_{\mathbf{w}}$ - PCI & corr. $_{\mathbf{w}} /$ In (Speed) - PCI \\
\hline Driver Seat & -0.9199 & -0.9226 \\
\hline Over the Central Axis & -0.9036 & -0.9037 \\
\hline Front Seat & -0.8305 & -0.8334 \\
\hline
\end{tabular}

\subsection{PCI Value Prediction}

At the present time, based on the availability of technological equipment, creating an acceleration measurement set with the intended precision is an easy task. Accelerometers can be found in many devices, including mobile phones, etc. and vibration data can be found through various programs. For this purpose, PCI value prediction models were developed by using vibration values that were obtained by measuring and evaluating at a single measurement point with an accelerometer with high sensitivity, calibration and reliability. 
At this point, it must be taken into consideration that measurements should be taken at a speed of $40 \pm 5 \mathrm{~km} / \mathrm{h}$, and the results of the evaluation should be corrected using calibration parameters produced on a calibration road which has the properties mentioned in the previous topics. In the last stage of the study, mathematical prediction models were created that use the corr. $a_{w}$ value produced from the vibration data measured at the driver's seat position and over the central axis. In all the model analyses, the results were accepted as statistically significant.

Firstly, after investigating the many different relationships between the PCI and the corr. $a_{w}$, produced during the measurement made at the driver's seat position, a linear model was created with a regression of $0.846(\mathrm{R}=0.920)$, which can give significant results to the data at the most suitable and the most extreme points. The created model is presented below. In here corr. $a_{w_{D S}}=$ corr. $a_{w_{\text {Driver Seat }}}\left(\mathrm{m} / \mathrm{s}^{2}\right)$ was shortened.

$$
\mathrm{PCI}=-184.276^{*} \text { corr. } \mathrm{a}_{\mathrm{wDS}}+96.002
$$

Table 4: corr. $\mathrm{a}_{\mathrm{w}} \mathrm{DS}$ - PCI estimate model statistical values.

\begin{tabular}{|l|c|c|c|c|}
\hline \multirow{2}{*}{} & \multicolumn{2}{|c|}{ Unstandardized Coeff. } & \multirow{2}{*}{$\mathrm{t}$} & \multirow{2}{*}{ Sig. } \\
\cline { 2 - 3 } & Coefficent & Std. Error & & \\
\hline Constant & 96.002 & 1.845 & 52.025 & 0.00 \\
corr. $\mathbf{a}_{\mathbf{w}}$ DS & -184.276 & 7.974 & -23.110 & 0.00 \\
\hline
\end{tabular}

Secondly, after investigating the many different relationships between the PCI and the corr. $a_{w}$ produced during the measurement over the central axis, a linear model was created with a regression of $0.816(\mathrm{R}=0.904)$, which can give significant results to the data at the most suitable and the most extreme points. The created model is presented below. In here, corr. $a_{w_{O C A}}=$ corr. $a_{w_{\text {Over the Central Axis }}}\left(\mathrm{m} / \mathrm{s}^{2}\right)$ was shortened.

$$
P C I=-116.773 * \text { corr. } a_{w_{O C A}}+92.305
$$

Table 5: corr. $\mathrm{a}_{\mathrm{w}}$ OCA- PCI estimate model statistical values.

\begin{tabular}{|l|c|c|c|c|}
\hline \multirow{2}{*}{} & \multicolumn{2}{|c|}{ Unstandardized Coeff. } & \multirow{2}{*}{$\mathrm{t}$} & \multirow{2}{*}{ Sig. } \\
\cline { 2 - 3 } & Coefficent & Std. Error & & \\
\hline Constant & 92.305 & 1.886 & 48.947 & 0.00 \\
corr.a $\mathbf{a}_{\text {w }}$ OCA & -116.773 & 5.622 & -20.771 & 0.00 \\
\hline
\end{tabular}

A linear model was created with a regression of $0.856(\mathrm{R}=0.925)$ between the PCI and the corr. $a_{w}$, along with the measurement speed that was calculated from the measurement made at the driver's seat position. In the model, speed = the average measurement speed of section $(\mathrm{km} / \mathrm{h})$ was shortened.

$$
P C I=-186.483 * \text { corr. } a_{w_{D S}}+0.948 *(\text { speed })+58.868
$$

Table 6: corr. $a_{w}$ DS and speed - PCI estimate model statistical values.

\begin{tabular}{|l|c|c|c|c|}
\hline \multirow{2}{*}{} & \multicolumn{2}{|c|}{ Unstandardized Coeff. } & \multirow{2}{*}{$\mathrm{t}$} & \multirow{2}{*}{ Sig. } \\
\cline { 2 - 3 } & Coefficent & Std. Error & & \\
\hline Constant & 58.868 & 14.496 & 4.061 & 0.000 \\
corr.a & & 7.798 & -23.914 & 0.000 \\
Speed & -186.483 & 0.367 & 2.582 & 0.011 \\
\hline
\end{tabular}

A linear model was also created with a regression of $0.817(\mathrm{R}=0.904)$ between the PCI and the corr. $a_{w}$, along with the measurement speed that was calculated from the measurement made over the central axis.

$$
P C I=-42.890 * \frac{\text { corr. } a_{w_{O C A}}}{\ln (\text { speed })}+92.343
$$


Table 7: corr. $a_{w}$ OCA and speed - PCI estimate model statistical values.

\begin{tabular}{|l|c|c|c|c|}
\hline \multirow{2}{*}{ Constant } & \multicolumn{2}{|c|}{ Unstandardized Coeff. } & \multirow{2}{*}{$\mathrm{t}$} & \multirow{2}{*}{ Sig. } \\
\cline { 2 - 3 } & Coefficent & Std. Error & & \\
corr.a $\mathbf{a}_{\mathbf{w}}$ OCA $/ \ln$ (Speed) & 92.343 & 1.886 & 48.957 & 0.00 \\
& -429.890 & 20.682 & -20.785 & 0.00 \\
\hline
\end{tabular}

As it is seen, the accuracies of predictions provided by the generated mathematical models are highly close to each other.

\section{Conclusions}

There have been many studies determining the high relationship between the structural performance of the pavement and driving comfort, and it is believed that the performance of the pavements can be determined from the vertical vibrations that occur in the vehicle, as the best reflection of driving comfort.

In this study, the difficult and arduous field works of the PAVER system that was used in determining the current performance of the pavement decreased to a minimum level and tried to be made usable by the units responsible for the management of the pavements particularly in urban roads.

This study provides an explanation of a developed measurement method, while also measurement speed of 40 $\mathrm{km} / \mathrm{h}$ for the measurement of the acceleration data that will be used in the evaluation of the vibration. The measurement speed has been chosen as $40 \pm 5 \mathrm{~km} / \mathrm{h}$ for urban roads.

As an outcome of this study, forecast models have been created that can predict PCI values from vibration data. The models developed using the regression method included PCI prediction models that take into account the measurement speed, driver seat cor. $\mathrm{a}_{\mathrm{w}}$ and over the central axis corr. $a_{w}$ components. These models permit the identification of relationships in which the regression value is very high between the dependent and the independent variables. It was determined that both the regression results of the models were statistically significant and their accuracy with respect to prediction are also rather high.

It has been thought that establishing a PMS at the network level for a HMA type highway network by a unit responsible for the management of the pavements by the help of this study and the models produced would be rather easy and functional. It is surely beyond doubt that it is necessary to undertake observations and measurements in place in order to generate maintenance and repair and renovation implementation projects for the sections within the framework of a PMS to be put into practice by the help of this study. Implementing the findings of this study in various regions and with various vehicles and making a comparison of the results will strengthen the accuracy of the models. To contribute to the practical implementation of the model, the findings of the study can be transformed into a package program that can be presented for use by the related institutions/enterprises.

\section{Acknowledgements}

This work was supported by the Scientific Research Projects Program of İstanbul University (Project No 21794).

\section{References}

[1] R. Haas, W. R. Hudson, J. P. Zaniewski, Modern pavement management. Malabar, Florida, USA: Krieger Pub. Co., 1994.

[2] ASTM E1889-97: Standard Guide for Pavement Management Implementation, West Conshohocken, PA: ASTM International, 2009.

[3] M. Y. Shahin, Pavement management for airports, roads, and parking lots. Springer: New York, 2005.

[4] C. L. Monismith, F. N. Finn, J. A. Epps, M. Kermit, Pavement management at the local government level. Transportation Research Board, 1987.

[5] I. Schacke, Pavement maintenance management systems in OECD countries. OECD, 1987.

[6] A. Wolters, K. Zimmerman, K. Schattler, A. Rietgraf, Implementing pavement management systems for local agencies, Research Report ICT-11-094-1, Illinois Center for Transportation, 2011.

[7] F. B. Holt, W. L. Gramling, Pavement management implementation. ASTM, 1991.

[8] Y. U. Shah, S. S. Jain, M. Parida, "Evaluation of prioritization methods for effective pavement maintenance of urban roads," International Journal of Pavement Engineering, vol. 15, no. 3, pp. 238-250, 2012.

[9] J. N. Meegoda, S. Gao, "Roughness progression model for asphalt pavements using long-term pavement performance data," Journal of Transportation Engineering, vol. 140, no. 8, 04014037, 2014. 
[10] L. Picado-Santos, A. Ferreira, A. Antunes, C. Carvalheira, B. Santos, M. Bicho, et al. Pavement management system for Lisbon. Thomas Telford Ltd., pp. 157-165, 2004.

[11] M. Karaşahin, S. Terzi, "Performance model for asphalt concrete pavement based on the fuzzy logic approach," Transport, vol. 29, no. 1, pp. 18-27, 2014.

[12] D. G. Lee, S.-K. Kim. "Impacts of geographical location and construction type on as-built roughness in highway pavement construction," KSCE Journal of Civil Engineering, vol. 9, no. 6, pp. 447-452, 2005.

[13] H. Ziari, J. Sobhani, J. Ayoubinejad, T. Hartmann, "Prediction of IRI in short and long terms for flexible pavements: ANN and GMDH methods," International Journal of Pavement Engineering, pp. 1-13, 2015.

[14] J.-D. Lin, J.-T. Yau, L.-H. Hsiao, "Correlation analysis between international roughness index (IRI) and pavement distress by neural network," in TRB $82^{\text {th }}$ Annual Meeting, Washington, 2003.

[15] K. A. Zimmerman, R. Knox, "Improving IDOT's pavement condition rating process," Journal of infrastructure systems, vol. 4, no. 2, pp. 79-85, 1998.

[16] J. H. Choi, T. M. Adams, H. U. Bahia, "Pavement roughness modeling using back-propagation neural networks," Computer-Aided Civil and Infrastructure Engineering, vol. 19, no. 4, pp. 295-303, 2004.

[17] U. Kırbaş, M. Karaşahin, "Performance models for hot mix asphalt pavements in urban roads," Construction and Building Materials, vol. 116, pp. 281-288, 2016.

[18] F. Wang, S. Easa, "Analytical evaluation of ride comfort on asphalt concrete pavements," Journal of Testing and Evaluation, vol. 44, no. 4, pp. 1671-1682, 2016.

[19] G. Cantisani, G. Loprencipe, "Road roughness and whole body vibration: evaluation tools and comfort limits," Journal of Transportation Engineering, vol. 136, no. 9, pp. 818-826, 2010.

[20] U. Kırbaş, M. Karaşahin, "Investigation of ride comfort limits on urban asphalt concrete pavements," International Journal of Pavement Engineering, pp. 1-7, 2016.

[21] A. González, E.J. O'Brien, Y. Y. Li, K. Cashell, "The use of vehicle acceleration measurements to estimate road roughness," Vehicle System Dynamics, vol. 46, no. 6, pp. 483-499, 2008.

[22] P. Múčka, "Road roughness limit values based on measured vehicle vibration," Journal of Infrastructure Systems, 04016029, 2016.

[23] K. L. Smith, L. Titus-Glover, L. D. Evans, Pavement smoothness index relationships, FHWA-RD-02-057, Final report FHWA:Champaign, IL 61820-3915 USA, 2002.

[24] K. Ahlin, N. O. J. Granlund, "Relating road roughness and vehicle speeds to human whole body vibration and exposure limits," International Journal of Pavement Engineering, vol. 3, no. 4, pp. 207-216, 2002.

[25] A. Bolling, J. Jansson, M. Hjort, M. Lidström, S. Nordmark, H.K. Sehammar, et al. "An approach for realistic simulation of real road condition in a moving base driving simulator," Journal of Computing and Information Science in Engineering, vol. 11, no. 4, 041009, 2002.

[26] R. Wix, S. Barlow, The Australian 3D Roughness Experience. ARRB Group Ltd, 2015.

[27] ASTM D6433-11: Standard Practice for Roads and Parking Lots Pavement Condition Index Surveys, West Conshohocken, PA: ASTM International, 2011.

[28] ISO 2631-1:1997(E): Mechanical vibration and shock - Evaluation of human exposure to whole-body vibration Part 1: General Requirement. Geneva, Switzerland: ISO, 1997. 